
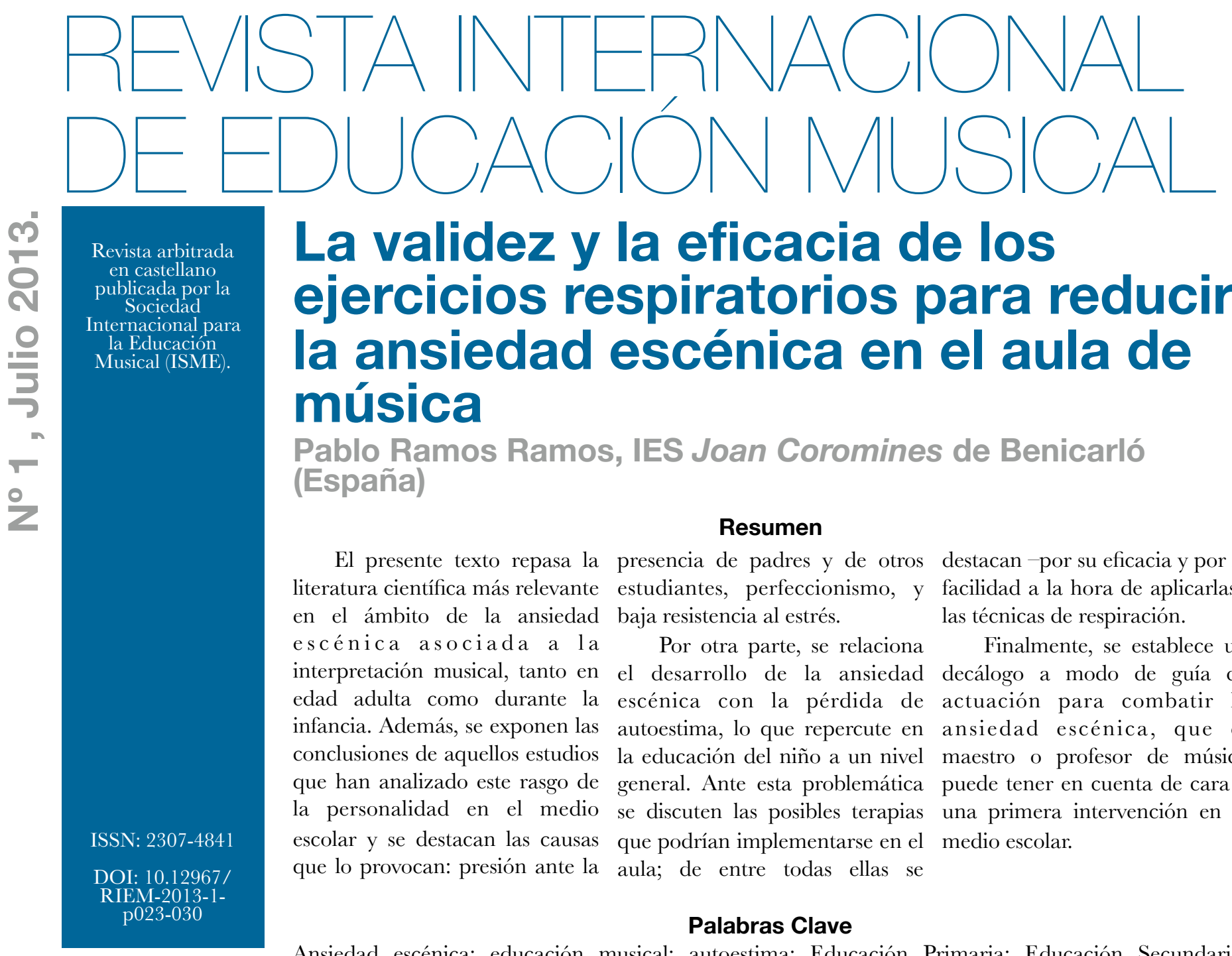

\title{
La validez y la eficacia de los ejercicios respiratorios para reducir la ansiedad escénica en el aula de música
}

\author{
Pablo Ramos Ramos, IES Joan Coromines de Benicarló \\ (España)
}

\section{Resumen}

El presente texto repasa la presencia de padres y de otros destacan-por su eficacia y por la literatura científica más relevante estudiantes, perfeccionismo, y facilidad a la hora de aplicarlasen el ámbito de la ansiedad baja resistencia al estrés.

escénica asociada a la Por otra parte, se relaciona las técnicas de respiración. interpretación musical, tanto en el desarrollo de la ansiedad decálogo a modo de guía de edad adulta como durante la escénica con la pérdida de actuación para combatir la infancia. Además, se exponen las autoestima, lo que repercute en ansiedad escénica, que el conclusiones de aquellos estudios la educación del niño a un nivel maestro o profesor de música que han analizado este rasgo de general. Ante esta problemática puede tener en cuenta de cara a la personalidad en el medio se discuten las posibles terapias una primera intervención en el escolar y se destacan las causas que podrían implementarse en el medio escolar. que lo provocan: presión ante la aula; de entre todas ellas se

\section{Palabras Clave}

Ansiedad escénica; educación musical; autoestima; Educación Primaria; Educación Secundaria; técnicas de respiración.

\section{Validity and Efficiency of Breath Exercises to reduce stage fright at the music classroom}

\section{Pablo Ramos Ramos, Benicarló High School Joan Coromines (Spain)}

\section{Abstract}

This paper reviews the most and other students, with this anxiety. Breath relevant literature in the field of perfectionism, and low resistance techniques are the most stage fright associated with to stress. important strategies because of musical performance, both in On the other hand, this their efficiency and ease of adulthood as in childhood. It paper relates the development of application.

also presents the findings of stage fright with the loss of self- Finally, a Decalogue is

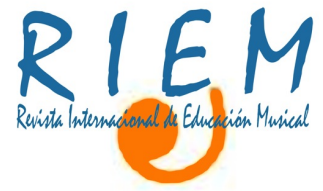

International Society for Music Education ISMEO Established in 1953 (2) studies that have analyzed this feature of personality at schools and highlights the reasons causing stage fright, such as: pressure in presence of parents esteem, which affects child's established as a guide for action education at large. Potential to combat performance anxiety therapies that could be which generalist and music implemented in the classroom teachers could take into account are discussed in order to cope ahead of a first play at schools.

\section{Palabras Clave}

Stage fright; music education; self-steem; Primary Education; Secondary Education; breath techniques. 


\title{
La validez y la eficacia de los ejercicios respiratorios para reducir la ansiedad escénica en el aula de música
}

\author{
por Pablo Ramos Ramos, IES Joan Coromines de Benicarló (España)
}

\section{La ansiedad a escena}

Experimentar ansiedad al enfrentarse a un público, ya sea conocido o desconocido, es algo inherente a la práctica musical. De la misma manera, muchas actividades, como la realización de exámenes y de ejercicios matemáticos, el hablar en público, la práctica deportiva o la interpretación en danza o arte dramático pueden generar en muchos individuos altos grados de ansiedad (Kenny, 2011).

En el ámbito anglófono se emplean diferentes términos para este fenómeno: stage frigth, performance anxiety, music performance anxiety... Como señala Kenny (2011), hay autores que consideran estos términos como diferentes grados de la ansiedad experimentada durante la interpretación musical. Otros, sin embargo, piensan que todos ellos son equivalentes. En las siguientes páginas adoptaremos esta segunda interpretación, en la que el término ansiedad escénica (AE) delimita una misma realidad que puede tener diferentes grados de intensidad. La ansiedad es algo normal e incluso útil -dentro de ciertos límites-, ya que permite mejorar el rendimiento en aquellas actividades en las que hay observadores. Sin embargo, la complejidad de este rasgo de la personalidad hace que sea muy difícil de controlar por parte del individuo, generando en ocasiones trastornos serios.

Spielberger (1972, Op. Cit. en Ortiz, 2009) fue el primer autor en definir con claridad los componentes de la ansiedad, diferenciando entre ansiedad rasgo y ansiedad estado. La primera es un rasgo relativamente estable de la personalidad que nos predispone para la actividad diaria; la segunda, corresponde a un estado emocional transitorio que aparece cuando el individuo interpreta como amenazante la situación a la que se enfrenta en ese momento. La AE surge como suma de la ansiedad rasgo de una persona más la ansiedad estado generada por el hecho de actuar en público, siendo ambas igual de determinantes. Se ha demostrado que personas con una ansiedad rasgo elevada tenían menor rendimiento en audiciones que personas de rasgo más bajo (Kenny, 2011).

$\mathrm{Si}$, como hemos dicho, la ansiedad en escena es inevitable y puede ser incluso beneficiosa para la interpretación, lo cierto es que, pasado un cierto grado, el músico puede experimentar "un miedo persistente y angustioso en un contexto público, así como una disfunción en las habilidades interpretativas, todo ello en un grado injustificado dados el entrenamiento, el nivel de preparación y las aptitudes individuales" (Kenny, 2006, p. 52).

En cuanto a las diferencias de género, las mujeres tienden a desarrollar patrones más acusados de miedo escénico que los hombres (Lewinsohn y otros, 1998), con respuestas fisiológicas diferentes, como una mayor frecuencia cardíaca (LeBlanc y otros, 1997). Sin embargo, y como veremos más adelante, esta problemática se vuelve más compleja cuando hablamos de niños (Ryan, 2000).
Las causas de la AE son varias y dependen en gran medida de las características de cada individuo. Barlow (2000) desarrolla, a partir de la teoría de las emociones, un modelo en el que define tres condicionantes básicos de la ansiedad: una vulnerabilidad general de tipo biológico -congénita-, una vulnerabilidad psicológica basada en experiencias tempranas en las que el individuo desarrolla una sensación de control en situaciones importantes y, finalmente, una vulnerabilidad específica en la que se asocia la ansiedad a un objeto o una situación determinados. A partir de la evolución e interacción de estos factores, la persona puede desarrollar un sentimiento de falta de control frente a situaciones presentes que actuaría como catalizador de la ansiedad (ibíd.). Además, el autor destaca que, en las experiencias desarrolladas durante la niñez, los padres, amigos o cuidadores pueden ser considerados como evaluadores de la actividad en un sentido negativo (ibíd.).

Este último factor exterior -la presión del entorno familiar y académico- puede generar un problema de orden interno. El perfeccionismo, cuando está generado por una motivación extrínseca - como el excesivo control paternopuede potenciar la ansiedad en escena (Stoeberg y Eismann, 2007). Así, las personas muy perfeccionistas -sobre todo aquellas que establecen objetivos que no son acordes a sus aptitudes- desarrollan una ansiedad muy pronunciada, además de miedo e insatisfacción (Flett y Hewitt, 2005). Estas personas "gastan mucha energía en el proceso de evaluación, desarrollan ideas rígidas sobre lo que constituye el fracaso y el éxito y perciben este último como un todo o nada" (Kenny, 2011, p.74).

A estas causas se suman otras muchas que, en definitiva, se pueden integrar en dos grupos diferentes: factores personales - perfeccionismo, baja autoestima, baja resistencia al estrés, poco autocontrol, etc.- y factores ambientales -tamaño de la audiencia, ausencia de partitura, presencia de padres, maestros o amigos, etc.-(Ryan, 2005).

\section{El miedo escénico en edad infantil}

Durante las últimas décadas, la mayor parte de los estudios sobre AE en música se han centrado en niveles preprofesionales -estudiantes de conservatorio- y profesionales -músicos de orquesta, cantantes y solistas-. Sin embargo, el análisis de este problema desde la perspectiva del niño no ha sido, hasta hace relativamente poco, una prioridad para la psicología.

Uno de los primeros estudios sobre ansiedad e interpretación musical en edad infantil fue el de Simon y Martens (1979). Estos autores compararon la ansiedad generada antes de la realización de dos tipos de actividades - unas deportivas y otras musicales- en niños de entre 9 y 14 años. La conclusión fue que el mayor grado de ansiedad se producía en los niños que tenían que interpretar un solo instrumental. 
Boucher y Ryan (2010) señalan que la ansiedad previa a la interpretación musical en público aparece desde los 3 años de edad, siendo esta menor en niños que ya tienen experiencia en situaciones similares. Por su parte, este último autor (Ryan, 1999, Op. Cit. en Ryan 2005), en un estudio con niños de entre 6 y 14 años, que integraban bandas escolares, concluyó que el mayor porcentaje de alumnos con alta ansiedad aparecía en sexto grado (12 años de edad), con un $50 \%$ del total de su promoción.

$\mathrm{Si}$ atendemos a factores ambientales, se ha confirmado que a mayor grado de familiaridad con el contexto en el que se desarrolla la actuación - escenario conocido y presencia de familiares entre el público- la ansiedad también disminuye (Boucher y Ryan, 2010). Los resultados de este tipo de estudios pueden tener una aplicación directa en el aula:

Si solo la familiaridad del niño con el lugar de la actuación reduce el impacto sobre la ansiedad [...] puede que un ensayo en el lugar de la actuación el día anterior fuera una manera simple, aunque efectiva, de mejorar el bienestar y el éxito del alumno durante el concierto (Íbid., p. 342).

Además, el tamaño de la audiencia y la percepción de la importancia de la actuación musical también condicionan el nivel de AE (LeBlanc y otros, 1997). La influencia del público sobre la ansiedad del niño también fue investigada por Ryan (1998), quien concluyó que su principal causa - en niños pianistas de 12 años- era el miedo a cometer errores de cara a otras personas, lo que repercutía, a su vez, en su autoestima.

De la misma manera, Passer (1983), en un estudio con niños de entre 10 y 15 años participantes en una liga de fútbol, destaca la presión que pueden sufrir estos al sentirse juzgados en su actividad por personas cercanas. Así, niños con una alta ansiedad rasgo en competición sienten como más vergonzosas las malas actuaciones -desde el punto de vista deportivo- ${ }^{-}$y piensan que recibirán un mayor nivel de crítica y desaprobación por parte de sus padres y de sus entrenadores.

Ryan (2004) evaluó la ansiedad frente a una audición de piano teniendo en cuenta el sexo del alumnado. Se midió la frecuencia cardíaca de los participantes en tres momentos diferentes -durante las clases normales, justo antes de subir al escenario y durante la audición-. Los resultados indicaron que las niñas desarrollan una mayor ansiedad anticipatoria, es decir, antes de subir al escenario, pero que los niños tienen una mayor frecuencia cardíaca una vez están en este. En las niñas se observó una relación directa entre el grado de ansiedad y la calidad de la interpretación. En los dos estadios analizados, los niños, a pesar de tener menor frecuencia cardíaca antes de subir al escenario, presentaban comportamientos más ansiosos desde el punto de vista de la actividad física. En este sentido, los niños con baja frecuencia cardíaca antes de subir al escenario y mucha actividad física interpretaban mejor que aquellos que tenían más frecuencia cardíaca y no tenían tantos comportamientos ansiosos, es decir, el movimiento les ayudaba en cierta manera a reducir la ansiedad. En este último punto -la relación entre frecuencia cardíaca, actividad motora e interpretación-, las chicas presentaron un comportamiento más homogéneo.

\section{La ansiedad y la autoestima en el aula de música}

La presión diaria de las clases colectivas de música

Aunque casi todos los estudios sobre AE en edad infantil se han centrado en la interpretación individual, muchas de sus conclusiones pueden ser extrapoladas a contextos de enseñanza colectiva. Sin embargo, existe una diferencia muy importante entre la enseñanza de un instrumento de forma individual y la asignatura de música en colegios e institutos, a saber: en esta última, el alumnado tiene una presión extra derivada del hecho de que sus cualidades musicales pueden ser juzgadas, ya no solo por el profesor, sino también por el resto de compañeros -en las clases individuales este último condicionante no es tan fuerte-- Así pues, cuando hablamos de $\mathrm{AE}$ en un aula numerosa debemos tener en cuenta que cada sesión es susceptible de convertirse en un pequeño examen para el alumnado; de ahí que el control diario de la ansiedad sea clave para un normal desarrollo de nuestra materia.

Desde el punto de vista del condicionamiento clásico, el miedo y la ansiedad durante la infancia pueden generarse tras varios episodios negativos y persistir mucho tiempo después (Field, 2006). Por ello, las experiencias negativas en el aula de música durante los primeros años de edad pueden influir de forma nefasta en la manera en la que los niños afrontan dicha asignatura durante la Educación Primaria e, incluso, durante la Secundaria.

Además de la natural presencia de ansiedad, el alumnado puede desarrollar patologías asociadas a esta. Sin embargo, como señala Kenny (2011), es difícil saber cuándo se está frente a un desorden serio, porque la interpretación musical en público puede conllevar altos grados de ansiedad. Por ejemplo, como indica el Diagnostic and statistical manual of mental dirorders, de la American Psychiatric Association (2000):

El miedo escénico y la timidez en situaciones sociales en

las que hay personas desconocidas es común y no debe ser diagnosticado como Fobia Social a menos que la ansiedad lleve a una incapacidad clínica significativa o genere angustia (p.323).

En este sentido, la aparición de un miedo escénico muy pronunciado puede llevar a la sospecha de que el alumno padece un trastorno asociado a la ansiedad -como el trastorno de ansiedad generalizado, el trastorno obsesivocompulsivo o la citada fobia social- que el psicólogo del centro puede detectar y tratar. No en vano, casi un tercio de los casos más pronunciados de AE han estado influidos por un desorden de ansiedad ya presente en el individuo (Sanderson, DiNardo, Rapee y Barlow, 1990, Op. Cit. en McGrath, 2012).

No obstante, la detección de estos problemas no siempre es fácil y el docente debe estar muy atento a las reacciones de su alumnado. En el caso de los adolescentes, la identificación de este tipo de conductas es más difícil, ya que estos no expresan de forma fluida sus sentimientos, sufriendo en silencio la ansiedad generada por las tareas escolares (Huberty, 2009).

En definitiva, podemos afirmar que la presencia diaria de un evaluador colectivo - los compañeros de clasedeterminan en gran medida la $\mathrm{AE}$ en el aula de música. A ello hay que sumar que la ansiedad se incrementa durante la realización de exámenes o audiciones, variando en grado 
según la percepción que tenga el alumno sobre la importancia de la prueba (Mitchell, 2011).

\section{La mejora de la autoestima}

Todo rasgo de la personalidad influye en el desarrollo global del niño y del adolescente y, a su vez, es influido por otros rasgos. En el caso de la ansiedad, la relación con la autoestima es evidente, la cual está conectada directamente con el desarrollo social del infante.

Como afirma Ryan (2000), es importante volver a evaluar las expectativas que tienen tanto el profesor como el alumno sobre la perfección de la interpretación -sobre todo en el ámbito escolar-, ya que incidir en el no hay que cometer errores lleva a una preocupación que aviva la ansiedad. Hembree (1988) analizó cómo esta búsqueda de perfección influye negativamente en la autoestima de los escolares, la cual está condicionada, a su vez, por las aptitudes, el sexo y la edad.

Tener una actitud más relajada frente a la interpretación musical en el aula ayuda al alumnado a aumentar su seguridad personal, lo que redunda en su desarrollo escolar y social. Como asegura Plummer:

Un niño que tiene un buen nivel de autoestima tiene más probabilidades de desarrollar todo su potencial y de generar relaciones satisfactorias que otro que sufre una acusada falta de autoestima [...]. [Este tipo de niños, además,] otorgan poco valor a sus habilidades y generalmente niegan sus éxitos; también encuentran dificultades a la hora de fijar sus objetivos y de resolver problemas. Algunos se dan por vencidos en ciertas tareas y, consecuentemente, rinden por debajo de sus aptitudes académicas y sociales (2001, p.15).

Además de hablar de autoconcepto y de autoestima, se debe tener en cuenta lo que Bandura (1977) definió como autoeficacia (Op. Cit. en Kenny, 2011). Este término se refiere a la confianza que tenemos en nuestras propias capacidades para conseguir resultados concretos. En este sentido, la autoeficacia influye decisivamente en la forma en la que una persona se enfrenta a la ansiedad. Sin embargo, la autoeficacia es algo muy específico (loc. cit.): una misma persona puede tener una autoeficacia muy alta en un área, pongamos por caso en lectura, y una muy baja en otra, como por ejemplo en música. Las terapias para reducir la $\mathrm{AE}$ permitirán al alumno desarrollar su concepto de autoeficacia, haciéndole ver que sus aptitudes - noqueadas por el miedoson mucho mayores de lo que piensa.

\section{¿Es posible una terapia en el aula?}

\section{El tratamiento de la ansiedad}

Reducir la AE es un proceso complejo que depende de la personalidad de cada individuo. Los métodos que se han venido utilizando para alcanzar este objetivo van desde el uso de drogas hasta la meditación, pasando por la respiración profunda, la técnica Alexander o la auto-hipnosis (Ryan, 2000).

Desde un punto de vista científico, existen dos tipos de tratamientos: los farmacológicos y los psicológicos. En el primer grupo hay que destacar los llamados bloqueadores beta, cuya principal virtud es mantener el ritmo cardíaco bajo y eliminar el temblor de las manos, todo ello sin alterar la capacidad de concentración. Sin embargo, estos deben estar prescritos por un médico, ya que pueden tener efectos adversos en pacientes con problemas cardíacos, diabetes o asma (Ryan, 2000). No hay que olvidar que dichos tratamientos se han desarrollado, entre otras cosas, para hacer frente a síntomas severos de la ansiedad y no para tratar el miedo escénico de forma generalizada.

El segundo tipo de métodos son de orden psicológico. Hembree (1988) afirma que estas terapias -entre las que encontramos la insensibilización sistemática, las técnicas de relajación, el modelado o la hipnosis- son eficaces a la hora de reducir la ansiedad infantil asociada a la realización de exámenes.

La insensibilización sistemática es el método más utilizado por los terapeutas -en pacientes con ansiedad, aunque no necesariamente AE. En este procedimiento cognitivo-conductual se entrena la relajación muscular a través de contextos de ansiedad inducida en los que se va aumentando poco a poco la intensidad. Nuestra opinión, no obstante, es que la insensibilización sistemática es un proceso demasiado complejo para ser implementado en el aula de música.

Por otra parte, las técnicas de relajación también son una terapia muy extendida a la hora de reducir la AE. Para conseguir este objetivo se desarrollan una serie de ejercicios que se centran en la relajación muscular y en los síntomas somáticos de la ansiedad. El proceso comprendería tres fases (McGrath, 2012): primero, distinción entre los estados de tensión y de relajación; segundo, detección de signos prematuros de tensión y cambios de comportamientos antes de que se desencadene la espiral de tensión; tercero, adquisición de técnicas que faciliten la relajación y permitan realizar la actividad con menos tensión.

En el ámbito estrictamente musical, la terapia que más ha arraigado en los últimos años es la técnica Alexander, en la que el alumno aprende una serie de estrategias para reducir las áreas de tensión del cuerpo, haciendo que los movimientos en el ejercicio de un instrumento -también la voz $^{-}$sean más naturales y requieran menos esfuerzo. A pesar del entusiasmo con en el que la comunidad musical ha acogido este sistema, su validez todavía no se ha demostrado con solidez. Únicamente existe una investigación científica al respecto: Valentine y otros (1995). Los resultados mostraron que la técnica Alexander podía ayudar a reducir la frecuencia cardíaca en momentos de estrés. Sin embargo, Kenny (2011) afirma que dicha investigación tiene un diseño muy débil y que sus conclusiones deben ser puestas en duda. En definitiva, a día de hoy no existiría una base científica sólida para afirmar que la técnica Alexander reduce los niveles de ansiedad durante la interpretación musical.

Existen muchas más terapias de tipo psicológico para combatir la $\mathrm{AE}$-como por ejemplo la retroacción biológica, la hipnosis o la terapia racional emotiva conductual (TREG), entre otras- no obstante, todas ellas requieren de especialistas muy bien formados para implementarlas. La lectura de la bibliografía más relevante sobre $\mathrm{AE}$ nos ha llevado a la conclusión de que hay pocas herramientas sencillas con las que combatirla, siendo las técnicas de respiración una de las más asequibles. Esta terapia puede permitir a un profesor de música, sin conocimientos exhaustivos de psicología, trabajar la $\mathrm{AE}$ en el medio escolar.

\section{Ejercicios respiratorios}

La forma en que respiramos influye en diversos procesos de nuestro cuerpo. Como señala McGrath (2012), la respiración diafragmática aumenta la cantidad de oxígeno en sangre y dota a los músculos de más energía. Por el contrario, la respiración superficial -habitual entre los músicos bajo 
estrés- dificulta el descenso de los niveles de ansiedad en el cuerpo.

Varios estudios han demostrado la capacidad de los ejercicios respiratorios a la hora de regular el sistema nervioso parasimpático -encargado de controlar las funciones involuntarias- desarrollando la concentración y la relajación (Chiang y otros, 2009; Dixhoorn, 1998; Güsewell, 2010).

La efectividad de las técnicas respiratorias se basa en una consecuencia somática de la ansiedad: la hiperventilación. Este fenómeno puede llevar asociados otros síntomas como el enfriamiento de las manos, el agarrotamiento de los dedos o dificultades en la respiración profunda, lo cual influye negativamente en la ejecución musical (Studer y otros, $\left.2011^{\mathrm{a}}\right)$.

Una de las primeras investigaciones sobre hiperventilación e interpretación musical (Widmer, Conway, Cohen y Davies, 1997) demostró la aparición de hiperventilación en músicos durante actuaciones con público. El estudio también concluyó que las mujeres desarrollan mayores niveles de $\mathrm{AE}$ y problemas de hiperventilación, teniendo el triple de probabilidad que los hombres de ser clasificadas -según el cuestionario Nijmegen ${ }^{1-}$ como hiperventilantes durante una actuación musical.

Studer y otros $\left(2011^{\mathrm{a}}\right)$ estudiaron el mismo fenómeno, pero asociándolo a la ansiedad anticipatoria, es decir, antes de la actuación. Este trabajo es sumamente importante, ya que para muchos músicos la ansiedad es mayor antes de la actuación que a lo largo de esta (Ibíd.). Las conclusiones establecieron una clara relación entre la hiperventilación y la $\mathrm{AE}$ en los momentos previos a la interpretación musical en público. Los autores dejaron abierta la posibilidad de que la AE y la hiperventilación fueran dos procesos que se refuerzan mutuamente, a saber: la primera acentuaría la segunda y viceversa (Ibíd.).

Esta última hipótesis se confirmaría en un estudio posterior (Studer y otros, 2012). En este se trabajó con estudiantes de música que tenían diferentes niveles de $\mathrm{AE}$, llegando la conclusión de que aquellos con altos niveles de AE desarrollaban una mayor hiperventilación: "estos hallazgos muestran, por primera vez, que el sistema respiratorio se activa antes de una actuación musical en público. El patrón respiratorio resultante está caracterizado por una respiración más irregular, ligeramente más torácica y creciente en intensidad" (p. 6).

Studer y otros $\left(2011^{\mathrm{b}}\right)$, en otra investigación con alumnado de conservatorio de grado universitario, mostraron que la técnica más utilizada por estos para combatir el miedo escénico eran los ejercicios respiratorios. El 54\% de los estudiantes que los había utilizado en alguna ocasión aseguró que eran muy efectivos, frente a un $45,1 \%$ que los definió como moderadamente efectivos y un $0,9 \%$ que no los consideró útiles.

Otros estudios han mostrado la validez de terapias que llevan asociados ejercicios respiratorios en la lucha con la $\mathrm{AE}$ en músicos, como el yoga (Khalsa y otros, 2009). Sin embargo, son pocas las investigaciones que han analizado este problema en niños y adolescentes. Cabe preguntarse, por tanto, si esta terapia sería efectiva en el medio escolar.

Deen (2000) desarrolló un estudio sobre la efectividad de los ejercicios respiratorios y de relajación diarios en jóvenes estudiantes de canto. El análisis estadístico reveló una reducción significativa de la $\mathrm{AE}$, siendo esta disminución más pronunciada en aquellos estudiantes que tenían pocos años de formación vocal o que poseían altos grados de ansiedad rasgo -esta última conclusión también fue extraída por Güsewell (2010)-. Extrapolando estas conclusiones a la educación obligatoria, los ejercicios respiratorios serían especialmente indicados para el alumnado de Educación Primaria y Secundaria que no tiene una formación vocal amplia.

La investigación que más luz arroja sobre nuestro objetivo - encontrar un método útil para reducir la ansiedad en el aula de música de la educación obligatoria- es el de $\mathrm{Su}$ y otros (2010). Este grupo de investigadores pusieron a prueba la efectividad de las técnicas de respiración y relajación en estudiantes de piano y violín, con edades comprendidas entre los 6 y los 12 años. Dicha terapia se realizó durante dos meses a razón de dos sesiones semanales, teniendo como objetivo el reducir la $\mathrm{AE}$ de cara a un recital con público al final de ese periodo. Se realizaron cuatro mediciones a lo largo del proceso: al comienzo (Q1), un mes antes de la prueba (Q2), cuando faltaban 30 minutos para esta (Q3) y, finalmente, cuando solo quedaban 5 minutos (Q4). Los resultados, en una escala que mide la ansiedad de 0 a 90, fueron los siguientes: 39,49 (Q1), 41,15 (Q2), 42,66 (Q3) y $39,53\left(Q^{4}\right)$.

Dos conclusiones surgieron con fuerza. La primera es que no existe una reducción a largo plazo de la $\mathrm{AE}$, ya que esta es creciente hasta el mismo día del examen. La segunda, y más importante para nosotros, es que estos ejercicios sí que reducen la ansiedad a corto plazo, ya que entre la mediciones Q3 y Q4 se realizó un último ejercicio de respiración -exactamente 10 minutos antes de subir al escenario-, lo que redujo considerablemente la ansiedad justo antes de la última medición. A pesar de que la ansiedad no disminuye a largo plazo, los autores justifican la realización semanal de estos ejercicios para desarrollar el hábito y la técnica de la respiración y la relajación para que, así, el alumno la pueda utilizar de forma autónoma. Esta idea también la defiende McGrath (2012), quien asegura que desarrollar rutinas de respiración profunda antes de las actuaciones puede ayudar al músico a conseguir una condición mental y física favorable a la hora de la interpretación en público.

\section{La importancia de los métodos sencilllos y eficaces}

Como ya hemos mencionado, Su y otros (2010) desarrollaron para su estudio un entrenamiento de relajación y respiración en el que el alumnado realizaba los ejercicios - de 10 minutos de duración- durante dos días a la semana y de forma colectiva. El profesor ponía una cinta que contenía, en un primer plano sonoro, las instrucciones de los ejercicios y, en un segundo plano, música relajante. Esto era lo que oía el alumnado:

Queridos alumnos: poned vuestros pies en el suelo, mantened la espalda contra el respaldo de la silla y encontrad una postura cómoda. Cerrad los ojos y empezad a inspirar y espirar lentamente. Utilizad la nariz para coger aire y sentid cómo se expande vuestro vientre. Usad la boca para soltar el aire. Recordad que no hay que aguantar la respiración y que el cuello, los hombros y las extremidades deben estar relajadas (p. 83).

Como podemos observar, este tipo de ejercicios contienen instrucciones sencillas. Güsewell (2010), en su investigación para reducir la $\mathrm{AE}$ en estudiantes de 
conservatorio, también destacó la necesidad de protocolos básicos: "estoy tumbado sobre la espalda. Pienso en mi pie izquierdo e imagino que mi respiración se dirige hacia él. Mi respiración se dirige ahora hacia los dedos del pie, después hacia el tobillo, la rodilla..." (p. 60).

Si las instrucciones son sencillas, los dos procesos que se pretenden activar con estos ejercicios no lo son menos: por un lado, el desarrollo de la respiración diafragmática y, por otro lado, el que el alumno sea consciente de su propia respiración. Así se llegará al estado de relajación necesario para reducir la $\mathrm{AE}$.

Como podemos observar, cualquier maestro o profesor puede desarrollar una terapia eficiente en el aula de forma fácil. Sin embargo, no es nuestra intención hacer una lista de los manuales o páginas web más indicadas para estos ejercicios, ya que en internet se puede encontrar material muy riguroso al respecto; es cuestión del docente el desarrollar un método adaptado a su aula. Los conceptos que le guiarán serán, como ya hemos dicho, muy sencillos: respiración diafragmática, toma de consciencia de la propia respiración y relajación.

\section{Conclusiones}

El presente texto ha pretendido arrojar un poco de luz sobre la problemática de la ansiedad escénica en el aula de Primaria y Secundaria. La herramienta que ofrecemos para tratarla es muy sencilla y pensamos que todo el profesorado de ambos niveles puede hacer uso de ella. En este sentido, proponemos un decálogo a tener en cuenta de cara a abordar este problema de forma autónoma:

1. La ansiedad escénica es algo inherente a la práctica musical y es nuestra obligación como profesores dotar al alumnado de herramientas eficientes para combatirla.

2. Todo rasgo de la personalidad influye en el desarrollo general del niño y del adolescente. Por tanto, el tratamiento de la AE no solo beneficiará el desarrollo de las clases de música, sino que se integrará de forma global en la educación del alumno.

3. Una AE muy pronunciada puede llevarnos a la sospecha de que estamos frente a un trastorno por ansiedad serio, que debe ser tratado por el psicólogo del centro.

4. El perfeccionismo, cuando está generado por una motivación extrínseca -como el excesivo control paterno- puede potenciar la ansiedad en el aula de música. En este caso, debemos hacer ver al alumnado que lo importante es el disfrute de la música en sí y no la evaluación.

5. El alumnado ve en los compañeros un elemento de observación y evaluación constante. Esta presión influye negativamente en su ansiedad, sobre todo en aquellos niños o adolescentes que tienen problemas de socialización.

6. Del anterior precepto se desprende que todas las clases colectivas de música pueden fomentar la $\mathrm{AE}$, por lo que esta se deberá tratar de forma habitual.

7. La terapia más accesible para tratar la AE son las técnicas de respiración. Su implementación no requiere de grandes conocimientos y son ejercicios que se adaptan muy bien al medio escolar.

8. Los ejercicios respiratorios son útiles en el corto plazo, es decir, se deben realizar, no de cara a una audición a semanas vista, sino al comienzo de cada sesión. Podemos dedicarle 10 minutos a modo de actividad introductoria.

9. Los aspectos a tener en cuenta para la relajación son: el empleo de la respiración diafragmática, la toma de conciencia de la propia respiración y la búsqueda de la relajación.

10. Cada profesor puede, con ayuda de internet y teniendo en cuenta las ideas expuestas anteriormente, desarrollar su propia metodología al respecto.

\section{Notas}

${ }^{1}$ El cuestionario Nijmegen mide el grado de hiperventilación. Para ello se establecen dieciséis preguntas en escala de intensidad en las que se evalúan sensaciones como dolor en el pecho, visión borrosa, respiración entrecortada, tensión alrededor de la boca, palpitaciones o respiraciones profundas pero muy rápidas, entre otras.

\section{Referencias}

American Psychiatric Association (2000). Diagnostic and Statistical Manual of Mental Disorders. Washington: American Psychiatric Association.

Bandura, A. (1977). Social Learning Theory. Englewood Cliffs: Prentice Hall.

Barlow, D. H., (2000). Unraveling the mysteries of anxiety and its disorders from the perspective of emotion theory. American Psychologist, 55(11), 1247-1263.

Boucher, H. y Ryan, C. A. (2011). Performance Stress and the Very Young Musician. Fournal of Research in Music Education, 58(4), 329-345.

Chiang L. C., Ma, W. F., Huang, J. L., Tseng, L. F., Hueh, K. C. (2009). Effect of relaxation-breathing training on anxiety and asthma signs/symptoms of children with moderate-to-severe asthma: A randomized controlled trial. International fournal of Nursing Studies, 46, 1061-1070.

Deen, D. (2000). Awareness and breathing: Keys to the moderation of musical performance anxiety. Dissertation Abstracts International, 60(12-A), 4241.

Dixhoorn, J. V. (1998). Cardiorespiratory effects of breathing and relaxation instruction in myocardial infarction patients. Biological psychology, 49, 123-135.

Field, A. P. (2006). Is conditioning a useful framework for understanding the development and treatment of phobias? Clinical Psychology Review, 26(7), 857-875.

Flett, G. L. y Hewitt, P. L. (2005). The perils of perfectionism in sports and exercise. Current Directions in Psychological Science, 14(1), 14-18.

Güsewell, A. (2010). Exercices respiratoires comme méthodes de gestion du trac -étude pilote. Recherche en Éducation Musicale, 28, 49-65. Disponible en http://rad.hemu.ch/ _multimedia/documents/reem_28_trac.pdf [recuperado el 14 de abril del 2012].

Hembree, R. (1988). Correlates, causes, effects and treatment of test anxiety. Review of Educational Research, 58(1), 44-77.

Huberty, T. J. (2009). Test and performance anxiety. Principal Leadership, 75(9), 12-16. Disponible en http:// www.nasponline.org/resources/principals / Anxiety_NASSP_Oct09.pdf [recuperado el 15 de abril del 2012].

Kenny, D. T. (2006). Music Performance Anxiety: Origins, phenomenology, assessment and treatment. Context, 31, 51-64. 
Kenny, D. T. (2011). The psychology of music performance anxiety. Nueva York: Oxford University Press.

Khalsa, S. B., Stephanie, M. S., Cope, S., Wyshak, G., y Sklar, E. (2009). Yoga Ameliorates Performance Anxiety and Mood Disturbance in Young Professional Musicians. Applied Psychophysiology and Biofeedback, 34, 279-289.

LeBlanc, A., Jin, Y. C., Obert, M., y Siivola, C. (1997). Effect of audience on music performance anxiety. Fournal of Research in Music Education, 45, 480-496.

Lewinsohn, P.M., Gotlib, I. H., Lewinsohn, M., Seeley, J. R., y Allen, N. B. (1998). Gender differences in anxiety disorders and anxiety symptoms in ado-lescents. Fournal of Abnormal Psychology, 107, 109-117.

McGrath, G. E. (2012). Music performance anxiety therapies: A review of the literature. Tesis doctoral. Urbana: Universidad de Illinois. Disponible en https:// www.ideals.illinois.edu/bitstream/handle/2142/31063/ McGrath_Casey.pdf?sequence=1 [recuperado el FECHA AQUí].

Mitchell, N. (2011). Evaluation and performance anxiety in music study. Canadian Music Educator, octubre, 32-34.

Ortiz, A. (2009). Music performance anxiety. A review of the literature. Tesis doctoral. Friburgo: Universidad de Fribuyrgo. Disponible en http://www.freidok.unifreiburg.de/volltexte/6603/pdf/MPA.pdf [recuperado el 4 de julio del 2012].

Passer, M. W. (1983). Fear of failure, fear of evaluation, perceived competence, and self-esteem in competitivetrait-anxious children. Journal of Sport Psychology, 5(2), 172-188.

Plummer, D. (2001). Helping children to build self-esteem. Londres: Jessica Kingsley.

Ryan, C. A. (1998). Exploring music performance anxiety in children. Medical Problems of Performing Artists, 13(3), 83-88.

Ryan, C. A. (1999). Musical performance anxiety: Are junior high band students affected? Opus, 41, 8-9.

Ryan, C. A. (2000). A study of the differential responses of male and female children to musical performance anxiety. Tesis doctoral. Montreal: Universidad McGill. Disponible en http://digitool.library.mcgill.ca/ [recuperado el FECHA AQUí].

Ryan, C. A. (2004). Gender differences in children's experience of musical performance anxiety. Psychology of Music, 32, 89-103.

Ryan, C. A. (2005). Experience of musical performance anxiety in Elementary school children. International fournal of Stress Management, 12(4), 331-342.

Sanderson, W. C., DiNardo, P. A., Rapee, R. M., y Barlow, D. H. (1990). Symptom comorbidity in patients diagnosed with DSM-III-R anxiety disorders. Fournal of Abnormal Psychology, 99, 308-312.

Simon J. A. y Martens, R. (1979). Children's Anxiety in Sport and Nonsport Evaluative Activities. Fournal of Sport Psychology, 1, 160-169.

Spielberger, D. C. (ed.) (1972). Anxiety: current trends in theory and research. Nueva York: Academic Press.

Stoeber, J. y Eismann, U. (2007). Perfectionism in young musicians: Relations with motivation, effort, achievement, and distress. Personality and Individual Differences, 43, 2182-2192.
Studer R. K., Danuser B., Hildebrandt H., Arial M., y Gómez P. (2011 $\left.1^{\mathrm{a}}\right)$. Hyperventilation complaints in music performance anxiety among classical music students. Fournal of Psychosomatic Research, 70, 557-564.

Studer R. K., Danuser B., Hildebrandt H., Arial M., y Gómez P. $\left(2011^{b}\right)$. Stage fright: Its experience as a problem and coping with it. International Archives of Occupational and Environmental Health, 84, 761-771.

Studer R. K., Danuser B., Hildebrandt H., Arial M., Wild P., y Gómez P. (2012). Hyperventilation in anticipatory music performance anxiety. Psychosomatic Medicine, 74(00).

Su Y. H., Luh J. J., Chen H. I., Lin C. C., Liao M. J., y Chen H. S. (2010). Effects of using relaxation breathing training to reduce music performance anxiety in 3rd to 6th graders. Medical problems of performing artists, 25(2), 82-86.

Valentine, E., Fitzgerald, D., Gorton, T., Hudson, J., y Symonds, E. (1995). The effect of lessons in the Alexander technique on music performance in high and low stress situations. Psychology of Music, 23(2), 129-41.

Widmer S., Conway A., Cohen S., y Davies P. (1997). Hyperventilation: A correlate and predictor of debilitating performance anxiety in musicians. Medical Problems of Performing Artists, 12(4), 97-106. 


\section{Sobre el Autor}

\section{Pablo Ramos Ramos}

Diplomado en Magisterio Musical por la Universidad de Valencia, realiza también estudios de Musicología en las Universidades de Poitiers (Francia) y Católica de Valencia, donde obtiene la licenciatura. Posteriormente, completa el Máster 2 en Investigación Musical de la Universidad de la Sorbona-París IV, bajo la dirección del Dr. François Madurell.

Como violinista se ha formado en el Conservatorio Municipal del Centro de París y en el Conservatorio Superior de Música de Castellón, donde obtiene el título superior de música.

Desde 2009 es profesor funcionario de enseñanza Secundaria de la Generalitat Valenciana. Actualmente desarrolla su labor docente en el IES foan Coromines de Benicarló (Castellón) e imparte talleres de música en el Instituto Valenciano de Atención a las Personas con Discapacidad y Acción Social (IVADIS) de la misma localidad.

Ha publicado artículos sobre música y educación en revistas como Revista Iberoamericana de Educación, Revista Complutense de Investigación en Educación Musical o Quadrivium, entre otras.

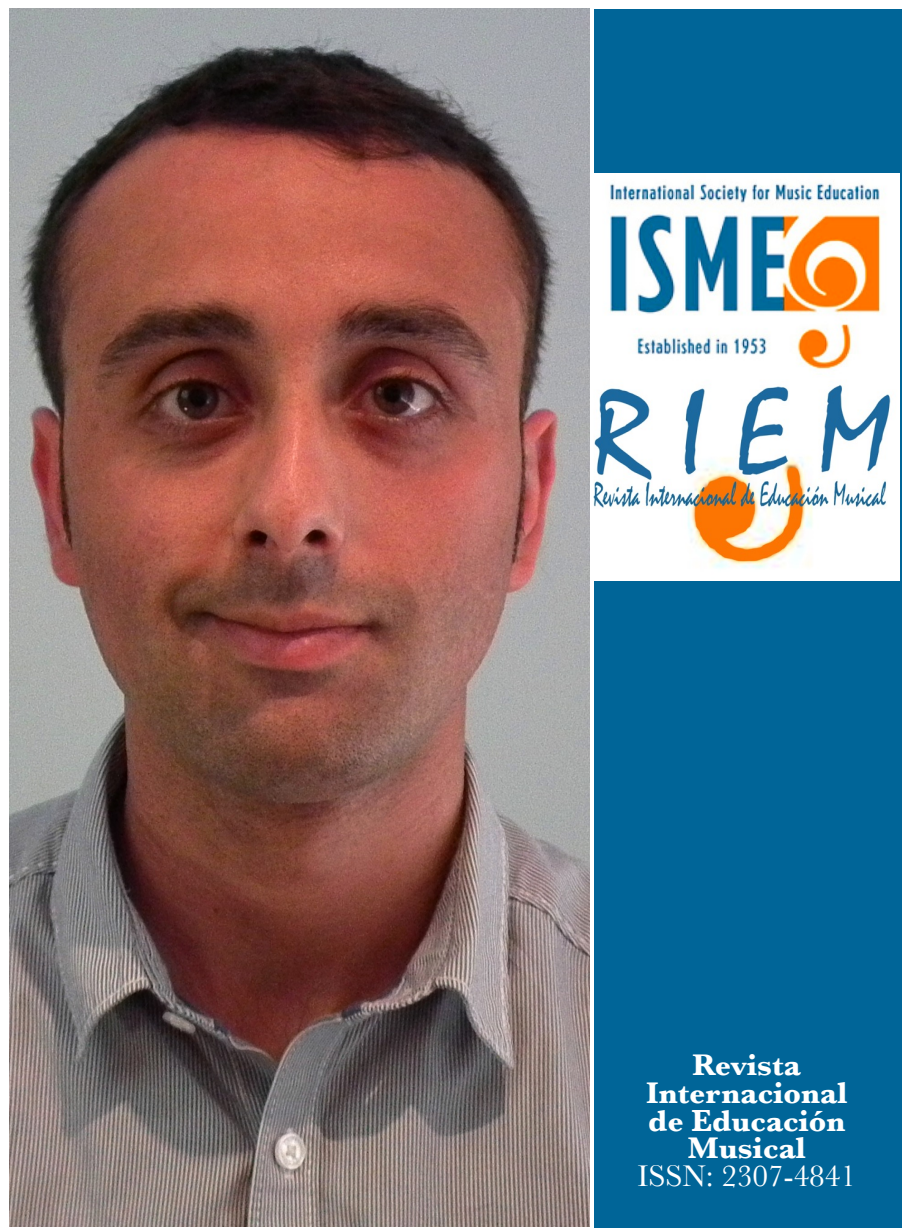

\section{EQUPO EDITORIAL}

Editor:

José Luis Aróstegui Plaza, Universidad de Granada (España)

\section{Editora Adjunta:}

Rosa María Serrano Pastor, Universidad de Zaragoza (España)

\section{Consejo Editorial}

María del Carmen Aguilar, Instituto Coral de Buenos Aires (Argentina)

Miquel Alsina Tarrés, Universidad de Gerona (España)

Graça Boal Palheiros, Instituto de Educación de Oporto (Portugal)

Rubén Gaztambide Fernández, Universidad de Toronto (Canadá)

Patricia Adelaida González, Universidad Autónoma de Chihuahua (México)

Claudia Gluschankof, Instituto Levinsky (Israel)

María Cecilia Jorquera Jaramillo, Universidad de Sevilla (España)

Gotzon Ibarretxe Txakartegi, Universidad del País Vasco (España)

Yore Kedem, Universidad de Illinois (Estados Unidos)

Saville Kushner, Universidad de XXX (Nueva Zelanda)

Ana Laucirica Larrinaga, Universidad Pública de Navarra (España)

Ana Lucia Louro, Universidad Federal de Santa María (Brasil)

Isabel Cecilia Martínez, Universidad Nacional de La Plata (Argentina)
Teresa Mateiro, Universidad del Estado de Santa Catarina (Brasil)

María Teresa Moreno, Universidad Laval (Canadá)

Graça Mota, Instituto de Educación de Oporto (Portugal)

Oscar Odena, Universidad de Glasgow (Reino Unido)

Gabriel Enrique Rusinek Milner, Universidad Complutense de Madrid (España)

Patricia Sabbatella Riccardi, Universidad de Cádiz (España)

Favio Shifres, Universidad Nacional de La Plata (Argentina)

Christopher Suazo, Colegio Americano de Madrid (España)

Maria dels Àngels Subirats Bayego, Universidad de Barcelona (España)

António Ângelo Ferreira Vasconcelos, Instituto de Educación de Setúbal (Portugal)

Gloria Patricia Zapata Restrepo, Universidad de Antioquía (Colombia) 